Irish Math. Soc. Bulletin

Number 80, Winter 2017, 45-68

ISSN 0791-5578

\title{
Aspects of Positive Scalar Curvature and Topology I
}

\author{
MARK G. WALSH
}

\begin{abstract}
Whether or not a smooth manifold admits a Riemannian metric whose scalar curvature function is strictly positive is a problem which has been extensively studied by geometers and topologists alike. More recently, attention has shifted to another intriguing problem. Given a smooth manifold which admits metrics of positive scalar curvature, what can we say about the topology of the space of such metrics? We provide a brief survey, aimed at the non-expert, which is intended to provide a gentle introduction to some of the work done on these deep questions.
\end{abstract}

\section{INTRODUCTION}

A central problem in modern geometry concerns the relationship between curvature and topology. A topological space may take many geometric forms. For example, while a sphere is usually thought of as round, one may alter its shape in various ways and still maintain the topological condition of being a sphere; see Fig.1. A large part of modern geometry therefore concerns the problem of finding a "good" geometry for some topological form, given a plethora of possibilities. By a good geometry, one may mean geometries with a particular property, concerning symmetry or curvature perhaps. Given such a geometric constraint, say constant curvature, the problem is to find examples of topological shapes which admit such geometries and to understand what the topological obstructions are in the ones that do not. For example, the round geometry is a constant curvature geometry on the sphere. It is also an example of a positive curvature geometry, the curvature itself being simply the reciprocal of the radius squared. A torus on the other hand can never be made to

2010 Mathematics Subject Classification. 46A99, 10A10.

Key words and phrases. Riemannian metrics of positive scalar curvature, spin manifolds, surgery, cobordism, Morse functions, loop spaces.

Received on 1-5-2017; revised 15-12-2017.

The author acknowledges support from Simons Foundation Collaboration Grant No. 280310. 
have everywhere positive curvature no matter how we deform it. This latter fact follows from the classic theorem of Gauss-Bonnet, a theorem we will return to very shortly.
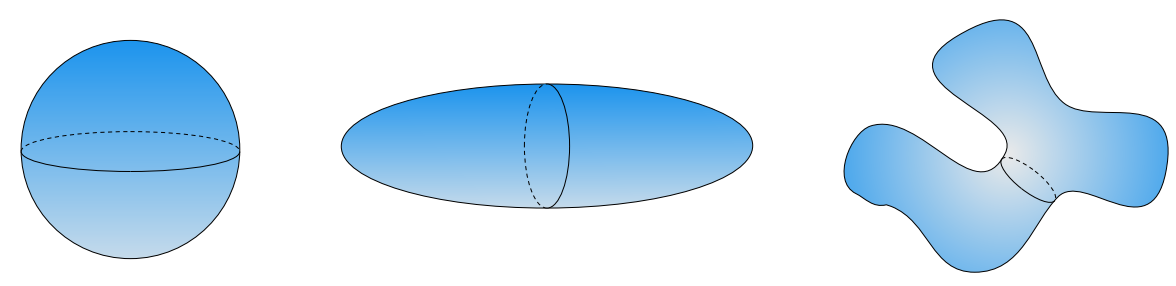

Figure 1. A selection of geometric structures on the sphere

Although there are a great many topological spaces one might consider, we will focus here on smooth compact manifolds. Usually, we are interested only in closed manifolds (those without boundary) although later we will discuss the case of manifolds with boundary. So what of the geometry? Given a smooth manifold $M$, of dimension $n$, the smooth structure on $M$ assigns to each point $x \in M$, an $n$ dimensional real vector space, $T_{x} M$, known as the tangent space to $M$ at $x$. These tangent spaces vary smoothly on $M$ and their disjoint union, $T M:=\bigsqcup_{x \in M} T_{x} M$, forms a vector bundle over $M$, known as the tangent bundle to $M$. It is possible to assign to each $x \in M$, a smoothly varying inner product $\langle\cdot, \cdot\rangle_{x}$ on $T_{x} M$. Such a choice of smoothly varying inner product is known as a Riemannian metric on $M$. It gives rise to a distance function (in the metric space sense) on $M$, but also allows for the measurement of angles and in particular, curvature. Any smooth manifold admits a multitude of possible Riemannian metrics each one determining a geometric structure on $M$. Once equipped with such a structure, the smooth manifold $M$ is said to be a Riemannian manifold. Although there are other types of metric beyond the Riemannian and other types of geometric structure beyond this, in this article we will regard a geometric structure on $M$ to be a choice of Riemannian metric.

In this context, the initial problem we mentioned can be restated as follows: given a smooth compact manifold $M$ and a geometric constraint, can we find a Riemannian metric on $M$ which satisfies this constraint? This is a very broad question and one which has motivated an enormous amount of research over the years. In this article, we will discuss this problem for one particular geometric constraint: positive scalar curvature. Thus, we consider the following 
question.

Question 1. Given a smooth compact manifold $M$, can we find a Riemannian metric, $g$, whose associated scalar curvature function, $s_{g}: M \rightarrow \mathbb{R}$, is strictly positive at all points on $M$ ? In other words, does $M$ admit a metric of positive scalar curvature (psc-metric)?

Before defining the scalar curvature function, or providing any justification as to why this geometric constraint is even interesting, let us consider a further question which is of significant current interest.

Question 2. In the case when $M$ admits a psc-metric, what can we say about the topology of the space of all psc-metrics on $M$ ? In particular, is this space path-connected? What about its higher homotopy or homology groups?

The first of these questions, the existence problem, has been studied extensively for several decades and a great deal is now understood on this matter. Much less is known about the second problem; however in recent years there have been some significant breakthroughs. The purpose of this article is to provide a brief survey on these problems which lie in an intriguing overlap of geometry, topology and analysis. This survey is not intended to be comprehensive. Such an undertaking would require the combined efforts of many experts and result in a voluminous article. Instead, it is intended to give the non-expert a taste of what is happening in a very interesting area of mathematics.

Acknowledgements. I am very grateful to Boris Botvinnik at the University of Oregon, and David Wraith and Anthony O'Farrell at Maynooth University, for their helpful comments and suggestions. Much of this article was written while I was an associate professor at Wichita State University. I would like to thank my former colleagues there for their friendship and support over the years.

\section{Why POSITIVE SCALAR CURVATURE?}

We begin with the case of a 2-dimensional Riemannian manifold $M$. The scalar curvature, $s: M \rightarrow \mathbb{R}$, is a smooth function which agrees up to a multiple of 2 with the classical Gaussian curvature, $K: M \rightarrow \mathbb{R}$. More precisely, $s=2 K$. Recall that, in the case when 
$M$ is obtained as an embedded submanifold of $\mathbb{R}^{3}$, the Gaussian curvature at any point $x \in M, K(x)$, is the product of the pair of principal curvatures at $x$. At this level, scalar curvature is a fairly intuitive concept. Round spheres have positive scalar curvature as principal curvatures have the same sign. Planes and cylinders have zero scalar curvature as at least one of the principal curvatures is zero. Finally, a saddle surface displays scalar curvature which is negative as principal curvatures in this case have opposite signs; see Fig.2. Importantly, this type of curvature is intrinsic to the surface itself and does not depend on the way the surface is embedded in Euclidean space. Hence, the flat plane and the round cylinder, both have the same flat scalar curvature, even though extrinsically they curve differently in $\mathbb{R}^{3}$.
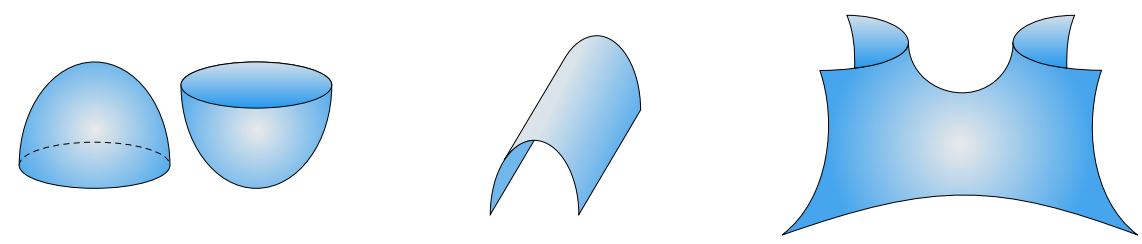

Figure 2. Two dimensional regions of positive, zero and negative scalar curvature

Geometrically then, positive scalar curvature can be thought to make a surface close in on itself whereas negative scalar curvature causes it to spread out. The topological consequences of this are evident from the classical theorem of Gauss-Bonnet. This theorem relates the scalar curvature $s$, of a compact oriented Riemannian 2-manifold $M$, with its Euler characteristic $\chi(M)$ by the formula

$$
\frac{1}{4 \pi} \int_{M} s=\chi(M)
$$

Recall that $\chi(M)$ is an integer obtained by the formula $\chi(M)=$ $v-e+f$ where $v, e$ and $f$ are respectively the numbers of vertices, edges and faces of a triangulation of $M$. That this formula is independent of the triangulation and gives a well-defined topological invariant is well-known. It follows that a closed surface with zero Euler characteristic, such as a torus, does not admit a metric of strictly positive (or negative) scalar curvature. Similarly, a surface with positive Euler characteristic such as a sphere cannot have scalar 
curvature which is everywhere non-positive. From the Uniformisation Theorem we know that every closed surface admits a metric of constant scalar curvature; see theorem 1.7 of [58]. This implies the following classification result: a closed surface admits a metric of positive, zero or negative scalar curvature if and only if its Euler characteristic is respectively positive, zero or negative.

In higher dimensions, the relationship between curvature and topology is much more complicated. The scalar curvature is one of three intrinsic curvatures which are commonly studied, the others being the Ricci and sectional curvatures. These curvatures vary greatly in the amount of geometric information they carry. The sectional curvature is the strongest and contains the most geometric information. At each point $x \in M$, where $M$ is a Riemannian manifold, the sectional curvature $K_{x}$ is a smooth real valued function on the space of all 2-dimensional subspaces of the tangent space $T_{x} M$. Each 2-dimensional subspace $V \subset T_{x} M$ is tangent to a locally specified 2-dimensional Riemannian submanifold of $M$. The sectional curvature $K_{x}(V)$ is simply the classical Gaussian curvature of this 2-dimensional Riemannian submanifold at $x$. The Ricci and scalar curvatures are successively weaker curvature notions and are obtained as follows. Let $\left\{e_{1}, e_{2}, \cdots, e_{n}\right\}$ be an orthonormal basis for $T_{x} M$. Assuming $i \neq j$, we define $K_{x}\left(e_{i}, e_{j}\right)$ to be the sectional curvature at the point $x$ of the plane spanned by $e_{i}$ and $e_{j}$. For each $i$, we define $\operatorname{Ric}_{x}\left(e_{i}\right)$ by the formula

$$
\operatorname{Ric}_{x}\left(e_{i}\right)=\sum_{j, j \neq i} K_{x}\left(e_{i}, e_{j}\right) .
$$

This extends linearly to specify a quadratic form on $T_{x} M$. Thus for some $v \in T_{x} M$, we define the Ricci curvature at $x$ in the direction $v$ to be $\operatorname{Ric}_{x}(v)$. Finally, we obtain the scalar curvature at $x, s(x)$ by the formula

$$
s(x)=\sum_{i} \operatorname{Ric}_{x}\left(e_{i}\right)=2 \sum_{i<j} K_{x}\left(e_{i}, e_{j}\right) .
$$

When we say, for example, that a Riemannian manifold $M$ has positive (zero, negative etc) sectional curvature, we mean that for every point $x \in M$ and every 2-plane $V \subset T_{x} M$, the number $K_{x}(V)$ is positive (zero, negative etc). The analogous statements for Ricci 
and scalar curvature are defined similarly. It follows from the successive averaging in the formulae above that conditions such as positivity or negativity of the sectional curvature necessarily hold for the subsequent Ricci and then scalar curvatures. The converse however is not true.

It is important to understand that conditions such as strict positivity or negativity of the sectional or even Ricci curvatures impose severe topological restrictions on the underlying manifold. For example, it follows from the theorem of Bonnet-Meyers that a Riemannian manifold whose Ricci curvature is bounded below by a positive constant, is necessarily compact and has finite fundamental group; see theorem 11.7 of [58]. The scalar curvature however, is a substantially weaker notion than the sectional or even Ricci curvatures. In particular, topological invariants such as the size of the fundamental group do not by themselves prevent positivity of the scalar curvature, unlike in the Ricci and sectional cases. In fact, given any compact manifold $M$, the product manifold $M \times S^{2}$, where $S^{2}$ is the two dimensional sphere, admits a psc-metric. This is because of the way the scalar curvature function splits over a product metric as a sum of the scalar curvatures on the individual factor metrics. By equipping the sphere with a round metric of sufficiently small radius, we can increase the curvature on the sphere factor to compensate for any negativity arising from $M$.

Given the extent of the averaging process in obtaining the scalar curvature, especially in high dimensions, it seems surprising that any geometric information survives at all. Interestingly, there is one piece of geometric information the scalar curvature does carry, concerning the volume growth of geodesic balls. In particular, the scalar curvature $s(x)$ at a point $x$ of an $n$-dimensional Riemannian manifold $M$, appears as a constant in an expansion

$$
\frac{\operatorname{Vol}\left(B_{M}(x, \epsilon)\right)}{\operatorname{Vol}\left(B_{\mathbb{R}^{n}}(0, \epsilon)\right)}=1-\frac{s(x)}{6(n+2)} \epsilon^{2}+\cdots,
$$

comparing the volume of a geodesic ball in $M$ with the corresponding ball in Euclidean space; see [34]. Thus, positive scalar curvature implies that small geodesic balls have less volume than their Euclidean counterparts while for negative scalar curvature this inequality is reversed. This, at least at the local level, coincides with our 
2-dimensional intuitions about the way surfaces respectively close in or spread out under positive or negative curvature.

We now return to the question which formed the title of this section: why positive scalar curvature? At this point, the reader may well ask: given its geometric weakness, why care about scalar curvature at all? And why positivity? Why do we not consider metrics of negative, non-negative or zero scalar curvature? Regarding the first question, in lower dimensions such as 2, 3 and 4, the scalar curvature is still geometrically quite significant. In particular, it plays an important role in general relativity; see for example chapter 3 of [7]. As for the second and third questions, a partial justification is that there are no obstructions to the existence of metrics of negative scalar curvature in dimensions $\geqslant 3$; see [60. Furthermore, any closed manifold which admits a metric whose scalar curvature is non-negative and not identically zero, always admits a metric of positive scalar curvature. This follows from the Trichotomy Theorem of Kazdan and Warner; see [52] and [53] (for a more thorough discussion of this matter, see section 2 of [76]). In the case of positive scalar curvature however, it is a fact that there are obstructions in dimensions $\geqslant 3$. Not all of these smooth compact manifolds admit Riemannian metrics of positive scalar curvature. This remarkable observation is discussed in the next section.

\section{The Existence Question}

We now focus on the first of the two questions posed in the introduction. When does a given smooth compact $n$-dimensional manifold admit a metric of positive scalar curvature? The case when the dimension $n=2$ is the classical situation described above and so the question is completely answered. Note that when $n=1$, the scalar curvature is not defined as one-dimensional manifolds have no intrinsic curvature. We therefore focus on the case when $n \geqslant 3$.

3.1. The Obstructive Side. In the early 1960s, André Lichnerowicz discovered an obstruction to the existence of positive scalar curvature metrics in the case of certain manifolds; see [59]. These manifolds, among other things, satisfy the condition of being spin. As the reader may gather from this term, the notion of spin manifold has strong connections to Physics. In fact, the fundamental formula used by Lichnerowicz to demonstrate this obstruction was actually derived independently by Erwin Schrödinger in 1932; see [82] (also 


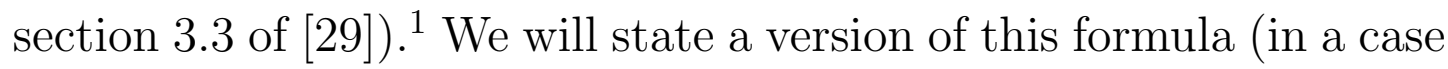
suitable for our purposes) shortly. Before doing so, we should say a few words about spin manifolds.

Spin, in the case of manifolds, is rather a technical notion to define. Here, we will provide only a casual description of one interpretation; for a detailed account the reader should consult [57]. Spin is essentially a strengthening of the condition of orientability and so we will begin by recalling what it means to say that a smooth manifold is orientable. Suppose on a smooth manifold $M$, we are given a point $x \in M$, a basis for $T_{x} M$ and a directed loop starting and ending at $x$. Let's assume for simplicity that the loop is an embedded circle. It is possible to continuously move the basis around the loop to obtain a new basis at $T_{x} M$ by a process called parallel translation. This is depicted in Fig. 3 below where the finishing basis is depicted with dashed lines. There are many ways to do this although a particularly nice one arises from a choice of Riemannian metric. We say that the manifold $M$ is orientable, if for any such quadruple of point, basis, loop and translation, the starting and finishing bases at $T_{x} M$ have the same orientation. Thus, the linear transformation of $T_{x} M$ which moves the starting basis to the finishing basis has positive determinant. Notice how this is not possible for certain loops on a Möbius band; see Fig4.

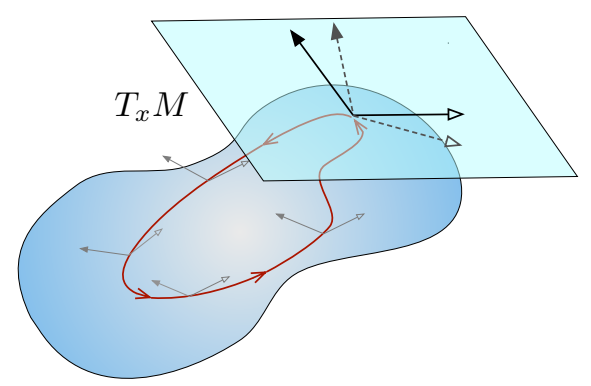

Figure 3. Translating a basis around a loop on an orientable manifold to obtain a new basis.

An equivalent formulation of orientability is as follows: the tangent bundle over any loop is trivial. That is, there is an isomorphism between $T M$ (restricted to the loop) and a product of the loop with $\mathbb{R}^{n}$. In particular, this necessitates that the normal bundle, $N$, the

${ }^{1}$ I am grateful to Thomas Schick for bringing to my attention Schrödinger's role in this story. 

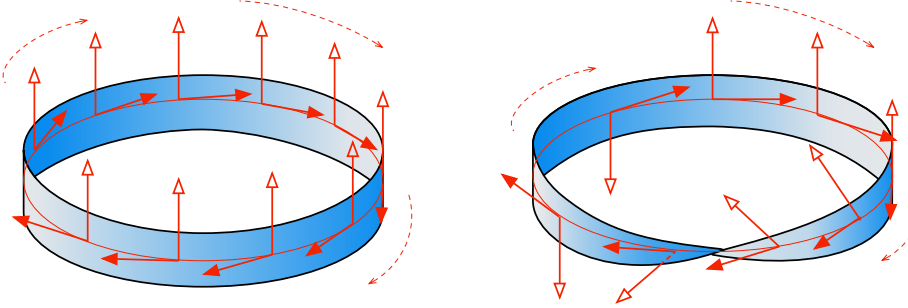

Figure 4. Comparing the translation of a basis around a loop on a cylinder and a Möbius band.

subbundle of $T M$ consisting of $(n-1)$-dimensional subspaces orthogonal to the loop, is also trivial (isomorphic to a product of the loop with $\mathbb{R}^{n-1}$ ). It is possible to identify this normal bundle with a small "tubular" neighbourhood of the loop. In the case of a loop with trivial normal bundle, the tubular neighbourhood is topologically just a product of the loop with the $(n-1)$-dimensional disk, $S^{1} \times D^{n-1}$. Thus, in the case of a two dimensional surface, a trivial normal bundle would correspond to a tubular neighbourhood which is, topologically, a regular cylindrical band of the type shown on the left of Fig.4. Loops whose tubular neighborhoods are Möbius bands correspond to loops with non-trivial normal bundles. A surface with such loops is therefore non-orientable. In fact, non-orientable surfaces are simply surfaces which contain Möbius bands.

In that it concerns loops, orientability can be thought of as a 1dimensional notion. Spin is in a sense a 2-dimensional strengthening of this. To simplify matters, let us assume that $M$ is a simply connected manifold with dimension $n \geqslant 5$. Simply connected manifolds are necessarily orientable. We now suppose we have an embedded two-dimensional sphere $S^{2} \subset M$, with $x \in S^{2}$. This embedded sphere can be thought of as a sort of "2-dimensional loop." The manifold $M$ is said to be spin if given any basis for $T_{x} M$, this basis can be extended continuously to obtain a family of bases over the embedded sphere. This determines a trivialisation of the tangent bundle restricted to the embedded sphere. Another interpretation (which is more useful for our purposes) concerns the normal bundle to the embedded sphere. A spin manifold is one for which every 
embedded 2-dimensional sphere has trivial normal bundle.2] This interpretation will be particularly relevant later on when we discuss the role of surgery.

Just as we may specify an orientation on an orientable manifold, on a spin manifold we may specify something called a spin structure. Let us suppose now that $M$ is a Riemannian spin manifold (with a specified spin structure). Such a manifold contains a wealth of interesting algebraic and geometric information. In particular, there exists a certain vector bundle over $M$ known as the spinor bundle. $3[\mathrm{We}$ will not describe the construction of the spinor bundle here, except to say that its construction involves, for each $x \in M$, replacing each tangent space $T_{x} M$ with a certain finite-dimensional complex vector space $S_{x}$ which is also a module over an algebra, known as the Clifford algebra of $T_{x} M$; see Part 1 of [3] for a readable introduction to Clifford algebras/modules. The construction of a Clifford algebra allows one to specify a geometrically significant multiplicative (algebra) structure on certain inner product spaces. When applied, for example, to $\mathbb{R}^{2}$ with the usual Euclidean dot product, it produces the quaternion algebra. In our case, Clifford algebras are associated, for each $x \in M$, to the tangent space $T_{x} M$ with multiplication arising ultimately from the inner product on $T_{x} M$ given by the Riemannian structure. This Clifford multiplication captures a great deal of information about the algebraic behaviour and symmetries of the curvature associated to the Riemannian metric. Put bluntly, it allows us to see things about the curvature which we would otherwise miss. We will return to this point in a moment.

Sections of the spinor bundle are known as spinor fields and so, denoting by $S \rightarrow M$ the spinor bundle itself, we consider the vector space $\Gamma S$ of all sections of this bundle. This is an infinite dimensional vector space, the construction of which is described in detail in [57]. On this vector space, one can define a certain first order linear differential operator, $D: \Gamma S \rightarrow \Gamma S$, known as the Dirac operator. Importantly, this operator is elliptic and self-adjoint. As the name suggests it was invented by the physicist Paul Dirac. One of the motivations behind the construction of this operator was a

\footnotetext{
${ }^{2}$ Without the hypothesis that the manifold is simply connected and of dimension at least 5, these interpretations do not quite work. See chapter 2 of [57] for a more thorough description.

${ }^{3}$ Spinors themselves are objects which are of great interest in Physics, describing intrinsic angular momentum or "spin" of subatomic particles.
} 
need to find a first order linear differential operator which squares as the Laplacian. In regular Euclidean space, this is exactly what happens. In our case, things are a little more complicated. The regular Laplacian is now replaced by an object known as the connection Laplacian on the spinor bundle $S \rightarrow M$. A connection on a smooth vector bundle is a means of differentiating sections of this bundle along tangent vector fields, something which is actually equivalent to being able to perform the parallel transport of vectors mentioned earlier. There are many choices of connection but in the case of a Riemannian manifold there is a particularly good choice of connection on the tangent bundle $T M \rightarrow M$, which best reflects the geometry of the underlying metric. This is the so-called Levi-Civita connection. In our case this leads, given the choice of spin structure, to a spinor bundle connection which allows us to sensibly differentiate spinor fields. Denoting this connection $\nabla$ and its adjoint (with respect to a certain global inner product on $\Gamma S$ arising from the Riemannian metric) by $\nabla^{*}$, we obtain the connection Laplacian $\nabla^{*} \nabla$. The Schrödinger-Lichnerowicz formula can now be stated as follows:

$$
D^{2}=\nabla^{*} \nabla+\frac{1}{4} s
$$

where $s$ is the scalar curvature function on $M$. Notice in this case that the scalar curvature is the obstruction to whether or not $D$ is truly a "square root of the Laplacian." Importantly, it is the role of the aforementioned Clifford multiplication which arises in the definition of $D$ that allows us to untangle the curvature information and see the right hand side of this equation.

So what does the Lichnerowicz formula tell us? If we assume that the scalar curvature $s: M \rightarrow \mathbb{R}$ is a strictly positive function, a fairly straightforward argument shows that the operator $D$ has trivial kernel. Thus, the existence of a non-trivial element of $\operatorname{ker} D$ (a so-called harmonic spinor field) implies that the Riemannian metric in question does not have positive scalar curvature. But this is only the beginning. It is a fact (although not a trivial one), that the kernel and cokernel of $D$ are both finite dimensional subspaces. In the case when the dimension of $M$ is a multiple of 4 , the spinor bundle splits into a certain pair of "even" and "odd" sub bundles $S_{+}$and $S_{-}$with a corresponding splitting of $D$ into $D_{+}$and $D_{-}$. Although $D$ is self-adjoint, implying that its kernel and cokernel have the same dimension, this is no longer the case with $D_{+}$or $D_{-}$. 
We now define the index of $D$, denoted ind $D$ as

$$
\operatorname{ind} D=\operatorname{dim} \operatorname{ker} D_{+}-\operatorname{dim} \operatorname{coker} D_{+} .
$$

It then follows that a necessary condition for the scalar curvature function to be positive is for the integer ind $D$ to be zero.

Notice however that we are still only dealing with a single Riemannian metric, the one used in the construction of $D$ in the first place. It is now we employ one of the great theorems of twentieth century mathematics: the Atiyah-Singer Index Theorem; see chapter III of [57] for a comprehensive discussion. This theorem equates the above index (which is an analytic index arising among other things from an individual choice of Riemannian metric) with a topological index for $M$. This topological index is known as $\hat{A}(M)$, the "A-hat"-genus of $M$. It depends only on the topology of $M$ and so is independent of the individual choice of Riemannian metric. This has a powerful consequence. If for any one Riemannian metric, the index ind $D$ above is computed to be non-zero, then it is non-zero for all choices of Riemannian metric. This is summarised in the following theorem.

Theorem 3.1. (Lichnerowicz [59])

Suppose $M$ is a closed spin manifold of dimension $n=4 k$ which admits a metric of positive scalar curvature. Then $\hat{A}(M)=0$.

The topological index $\hat{A}$ is therefore, in the case of closed, spin manfolds of dimension $4 k$, an obstruction to the existence of positive scalar curvature metrics. There are a great many closed spin manifolds for which this index is non-zero and so many examples of manifolds which do not admit psc-metrics. Consequently, such manifolds admit no metrics of positive Ricci or sectional curvature either.

In the 1970s, Hitchin showed how to generalise this index obstruction to other dimensions (beyond those divisible by 4); see [43]. Before saying anything further on this, it is worth briefly digressing to introduce a concept which plays a vital role in this story: cobordism. Two closed $n$-dimensional manifolds, $M_{0}$ and $M_{1}$, are said to be cobordant if there exists an $(n+1)$-dimensional manifold, $W$, with boundary $\partial W$ so that $\partial W=M_{0} \sqcup M_{1}$. Thus, the boundary of $W$ is a disjoint union of $M_{0}$ and $M_{1}$; see Fig.5. An elementary example of a pair of cobordant manifolds is the 2-dimensional sphere and torus. To see this, imagine a solid bagel with a round 
bubble trapped inside. The inner boundary is the sphere while the outer-boundary is the torus. Cobordism is an equivalence relation on closed $n$-dimensional manifolds and the set of equivalence classes actually forms a group, denoted $\Omega_{n}$, under the operation of disjoint union. In fact the collection, $\Omega_{*}=\left\{\Omega_{n}\right\}_{n \in \mathbb{N} \cup\{0\}}$, forms a graded ring under the operation of cartesian product of manifolds. There are more refined versions of cobordism, such as oriented cobordism, denoted $\Omega_{*}^{S O}$, and spin cobordism, denoted $\Omega_{*}^{\text {Spin }}$. In each case, two closed oriented (spin) manifolds are said to be oriented (spin) cobordant if their disjoint union forms the boundary of an oriented (spin) manifold with a consistent orientation (spin structure).

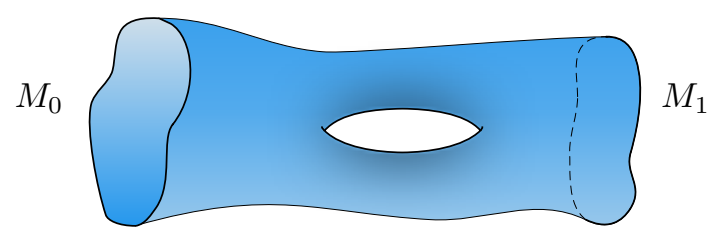

Figure 5. Two cobordant manifolds, $M_{0}$ and $M_{1}$

There is a graded ring homomorphism, defined by Milnor in [68], which takes the following form:

$$
\alpha: \Omega_{*}^{\text {Spin }} \longrightarrow K O_{*} .
$$

The target of this homomorphism is the real K-homology of a point, something we will not define here, except to say that it satisfies the following periodic isomorphism conditions.

$$
K O_{n} \cong\left\{\begin{array}{cl}
\mathbb{Z} & \text { if } n \equiv 0 \quad \bmod 4 \\
\mathbb{Z}_{2} & \text { if } n \equiv 1,2 \bmod 8 \\
0 & \text { otherwise }
\end{array}\right.
$$

This periodicity is an example of a phenomenon known as Bott periodicity, named after Raoul Bott, who first observed this behaviour in his study of the (stable) higher homotopy groups of certain classical Lie groups; see [9]. The homomorphism itself is surjective and moreover, when $n=4 k, \alpha$ and $\hat{A}$ coincide (at least up to a multiple of 2). Hitchin showed, in [43], that if $M$ is a closed spin manifold admitting a psc-metric, then $\alpha([M])=0$. Thus, a necessary condition for a closed spin manifold to admit a psc-metric is that it lies in a spin cobordism class which is in the kernel of $\alpha$. Establishing 
circumstances under which this is a sufficient condition (in general it is not) is the subject of the next section.

One fascinating consequence of Hitchin's work concerns exotic spheres. An exotic sphere is a smooth manifold which is homeomorphic to but not diffeomorphic to a standard sphere. Thus, an exotic sphere is topologically the same as, but smoothly different from, a standard sphere. Such objects were first constructed by Milnor in 1953, causing quite a stir in the mathematical community; see [66]. Hitchin showed that, starting in dimension 9, there are exotic spheres which admit no psc-metrics. In other words, the smooth structure on such a sphere is sufficiently exotic as to prevent positivity of even the weakest curvature. Given that topologically the sphere is the manifold most suited to positive curvature, this is an extraordinary fact.

We close this section by pointing out that the $\alpha$-invariant is not the only known obstruction to positive scalar curvature. Using certain minimal hypersurface methods of Schoen and Yau, described in [80], Thomas Schick constructed examples of spin manifolds in dimensions 5 through 8 with trivial $\alpha$-invariant and yet admitting no psc-metrics. These manifolds all have non-trivial fundamental group. Hence, the fundamental group of a manifold can, when coupled with other restrictions, be an obstruction. A much simpler example of this, following from work of Schoen and Yau in [81], is the fact that any 3-dimensional manifold whose fundamental group contains the fundamental group of a surface with positive genus, admits no psc-metric. Thus, the 3 -torus, $T^{3}=S^{1} \times S^{1} \times S^{1}$ admits no psc-metric, despite being a spin manifold and satisfying $\alpha\left(\left[T^{3}\right]\right)=0$. Indeed, the $n$-torus (the $n$-fold product of circles), $T^{n}=S^{1} \times \cdots \times S^{1}$ admits no psc-metric; see [36]. The situation in dimension 3 is now completely understood, since Perelman's proof of Thurston's Geometrisation Conjecture. The classification, which is discussed in the introduction to [63], is as follows: a closed 3-dimensional manifold admits a metric of positive scalar curvature if and only if it takes the form of a connected sum (see the next section for a definition) of spherical space forms (certain quotients of the round 3-sphere by actions of particular groups of isometries) and copies of $S^{2} \times S^{1}$. There is also a specific obstruction in the case of manifolds of dimension 4 arising in Seiberg-Witten theory; see [85]. Interestingly, it follows that there are examples of 4-dimensional manifolds which 
are simply-connected, spin and with $\hat{A}=0$, but which admit no psc-metric.

There is still a great deal we do not know about obstructions to positive scalar curvature. Consider for example, the case of nonsimply connected totally non-spin manifolds of dimension at least five. A totally non-spin manifold is one whose universal cover is non-spin and is, therefore, itself a non-spin manifold. This is a very large class of manifolds and it seems that there should be some obstructions here to positive scalar curvature. There are some individual examples of such manifolds which do not admit psc-metrics. In particular, Schoen and Yau have shown that the totally non-spin manifold obtained by taking a connected sum of $T^{6}$ with the product $\mathbb{C} P^{2} \times S^{2}$ (of 2-dimensional complex projective space with the 2 -sphere), admits no such metric. However, although some experts have made conjectures about this problem (see for example Stanley Chang's discussion of this matter in [20]), we have as yet nothing remotely analogous to the $\alpha$-invariant here.

3.2. The Constructive Side. The other side of the existence question concerns the problem of constructing examples of psc-metrics on manifolds where no known obstructions exist. The principle tool for doing this is known as the Surgery Theorem. This theorem was proved in the late 1970s by Gromov and Lawson [37] and, independently, by Schoen and Yau [80], and provides an especially powerful device for building positive scalar curvature metrics. A $p$-surgery (or codimension $q+1$-surgery) on a manifold $M$ of dimension $n$ is a process which involves removing an embedded sphere-disk product $S^{p} \times D^{q+1}$ and replacing it with $D^{p+1} \times S^{q}$, where $p+q+1=n$. The result of this is a new $n$-dimensional manifold $M^{\prime}$ whose topology is usually different from that of $M$; see Fig.6. For example, the 2dimensional torus can be obtained from the sphere via a 0 -surgery. Importantly, surgery preserves the cobordism type of the original manifold. Thus, if $M^{\prime}$ is obtained from $M$ by surgery, then $M$ and $M^{\prime}$ are cobordant and there is a complementary surgery which returns $M^{\prime}$ to $M$. The Surgery Theorem for psc-metrics can now be stated as follows.

Theorem 3.2. (Gromov-Lawson [36], Schoen-Yau [80])

Suppose $M$ is a smooth manifold which admits a metric of positive scalar curvature. If $M^{\prime}$ is a smooth manifold obtained from $M$ by 
a surgery in codimension at least three, then $M^{\prime}$ admits a metric of positive scalar curvature.

Note that a well-known example of a 0-surgery is the connected sum construction. Here, we remove a product $S^{0} \times D^{n} \cong D_{1}^{n} \sqcup D_{2}^{n}$ (a pair of disks) from a pair of disjoint $n$-manifolds $M_{1}$ and $M_{2}$, where $D_{1}^{n} \subset M_{1}$ and $D_{2}^{n} \subset M_{2}$. We then connect the resulting boundaries via a tube $[0,1] \times S^{n-1}$ (as suggested in Fig.66) to obtain the connected manifold $M_{1} \# M_{2}$, the connected sum of $M_{1}$ and $M_{2}$. With this in mind it is worth stating the following easy corollary of the Surgery Theorem.

Corollary 3.3. Suppose that $M_{1}$ and $M_{2}$ are $n$-dimensional manifolds, each admitting a metric of positive scalar curvature, and with $n \geqslant 3$. Then the manifold $M_{1} \# M_{2}$ obtained as a connected sum of $M_{1}$ and $M_{2}$ also admits a metric of positive scalar curvature.

In a moment we will discuss the implications of the Surgery Theorem. But first we will make a few comments about its proof. The proofs of this theorem by Gromov-Lawson and Schoen-Yau are quite different. The latter authors make use of PDE and certain minimal hypersurface methods to demonstrate the existence of psc-metrics on $M^{\prime}$. The Gromov-Lawson method is more obviously constructive. ${ }^{4}$ They begin with an arbitrary psc-metric, $g$ on $M$, and perform an explicit geometric construction of a new psc-metric, $g^{\prime}$ on $M^{\prime}$. This construction involves showing that, in a neighbourhood of the sphere to be removed by surgery, the psc-metric $g$ can be replaced by a psc-metric on $S^{p} \times D^{q+1}$ which is standard near $S^{p} \times\{0\}$. By this we mean the standard product of a round sphere with a "torpedo" shaped hemisphere. The difficulty is in adjusting the metric near the boundary of the region $S^{p} \times D^{q+1}$ to ensure a smooth transition back to the original metric while maintaining positive scalar curvature. This is where the codimension $\geqslant 3$ hypothesis comes in to play. It means that $q \geqslant 2$ and so the radial spheres making up the disk factor have dimension at least 2 , and therefore have some scalar curvature. Provided we are sufficiently close to $S^{p} \times\{0\}$, these radial spheres behave like round spheres and so, for small radius, have

\footnotetext{
${ }^{4}$ It was pointed out by Bernhard Hanke at a recent Oberwolfach workshop that many of us working in this field do not pay enough attention to the methods employed by Schoen and Yau here and work almost exclusively with the GromovLawson version of this theorem. This may result in some missed opportunities.
} 
large positive scalar curvature. This is then used to compensate for any negative curvature arising from the standardising adjustment. Once this standardised psc-metric is obtained, it is trivially easy to replace its standard torpedo shaped ends with a standard "handle" and complete the surgery, as shown in Fig.6.
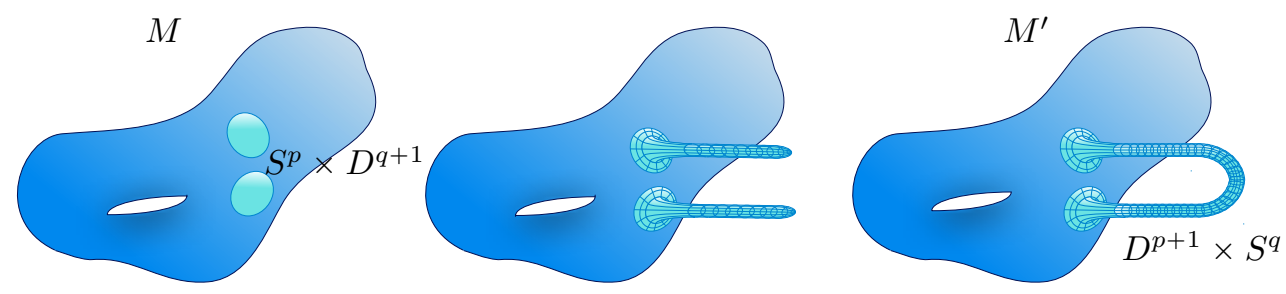

Figure 6. The original metric on $M$ (left), the metric after standardization on the region $S^{p} \times D^{q+1} \subset M$ (middle) and the post-surgery metric on $M^{\prime}$, after attachment of the handle $D^{p+1} \times S^{q}$ (right)

The effect of this theorem was to increase enormously the number of known examples of manifolds admitting psc-metrics. Suppose $M$ is a manifold which admits a psc-metric. It now follows that every manifold obtained by an appropriate surgery from $M$ also admits a psc-metric. It is worth pointing out that this theorem does not hold for positive Ricci or sectional curvature, as these curvatures do not exhibit the same flexibility as the scalar curvature. Positive scalar curvature however is sufficiently resilient as to be able to withstand significant topological adjustment.

To better understand this implication, let us consider the non-spin and spin cases separately. To avoid some other possible obstructions, let us further restrict our search to manifolds which are simply connected and of dimension at least 5 . In the non-spin case, no other obstructions were known. In [36], Gromov and Lawson went on to show that in fact there is no obstruction here. Every simply connected non-spin manifold of dimension $n \geqslant 5$ admits a psc-metric. This was done by considering the ring of oriented cobordism classes, $\Omega_{*}^{S O}$ and using the fact that this ring is generated by classes containing representatives built from complex projective space. These generating manifolds are non-spin and known to admit psc-metrics. Now, simply-connected manifolds are all orientable and every simply connected manifold is oriented cobordant to one of these known examples. Thus, given an arbitrary simply-connected manifold, there 
is a finite sequence of surgeries (though not necessarily in codimension $\geqslant 3$ ) which turn one of these known examples into our arbitrary manifold. Provided the arbitrary manifold is of dimension at least 5 and also non-spin, a topological argument shows such surgeries can be assumed to be in codimension at least three. The Surgery Theorem then allows us to use the psc-metric on the known example to construct one on the arbitrary manifold.

So what of the spin manifolds? We know from the work of Hitchin, that in looking for spin manifolds which admit psc-metrics we should restrict our search to spin cobordism classes of manifolds in the kernel of $\alpha$. But is there another obstruction here? Without added restrictions on dimension and the fundamental group the answer is yes. However, in analogous fashion to the non-spin case, Gromov and Lawson show in [36] that, provided we restrict to simply connected manifolds of dimension at least 5 , it is possible that no such obstruction exists. In particular, they show that (in these dimensions) it is enough to construct a collection of manifolds admitting psc-metrics which represent generating classes for the kernel of $\alpha$, since each simply connected spin manifold of dimension at least 5 which lies in such a class is obtainable from such a representative by codimension $\geqslant 3$ surgeries. An important step here is the removal from a spin manifold, by surgery, of certain topologically significant embedded 2-dimensional spheres. The spin condition, as interpreted in the previous section, implies that such embedded spheres have trivial normal bundles and so surgery is possible.

The task of constructing such a collection of representative manifolds, whose spin cobordism classes generate the kernel of $\alpha$, was finally completed by Stolz in [83]. Stolz showed that each generating class could be represented by a manifold which is the total space of a fibre bundle with quaternionic projective space fibres. The fibre of such a bundle admits a standard psc-metric and it is possible to construct a metric on the total space which restricts on fibres to this standard metric. Using well known curvature formulae due to O'Neill (see chapter 9 of [7]), it follows that the total space metric can be made to have positive scalar curvature by appropriately scaling the fibre metric. In summary we have the following theorem, known as the classification of simply connected manifolds of positive scalar curvature. 
Theorem 3.4. (Gromov-Lawson [36], Stolz [83]) Let $M$ be a compact simply connected manifold of dimension $n \geqslant 5$. Then $M$ admits a metric of positive scalar curvature if and only if $M$ is either not spin or $M$ is spin with $\alpha([M])=0$.

In the non-simply connected case, the problem of deciding which manifolds admit psc-metrics is ongoing. For certain types of fundamental group, this is the subject of a conjecture known as the Gromov-Lawson-Rosenberg Conjecture. Various analogues of this conjecture exist, which due to the discovery of counter-examples (most famously by Thomas Schick in [78]), have been reformulated a number of times. The statement of this conjecture is quite complicated and so we refer the reader to the following thoughtful surveys on these matters: [76], [79].

\subsection{The Existence Question for Manifolds with Boundary.} So far we have considered only the case of closed manifolds. It is important to mention that there are analogous problems for manifolds with boundary. Suppose $W$ is a smooth compact $(n+1)$ dimensional manifold with boundary, $\partial W=M$, an $n$-dimensional closed manifold. Without any further restrictions, the question of whether or not $W$ admits any psc-metrics is actually not such an interesting question. It turns out that, without some condition on the boundary, $W$ will not only always admit a metric of positive scalar curvature, but will in fact admit a metric of positive sectional curvature! This is a result of Gromov, see Theorem 4.5.1 of [35]. However, with appropriate boundary conditions, the question becomes extremely interesting. We consider only Riemannian metrics on $W$ which, near the boundary of $W$, take the form of a cylindrical product metric $\left(M \times[0,1], g+d t^{2}\right)$, where $g$ is some Riemannian metric on $M$ and $d t^{2}$ is the standard metric on the interval. We then consider the following question.

Question 3. When does a given psc-metric on $M$ extend to a pscmetric on $W$ which takes a product structure near the boundary?

Such extensions are not always possible. Indeed, as we will see in the next section, a better understanding of this problem would greatly help in our efforts to answer the second of the motivational questions posed in the introduction. 


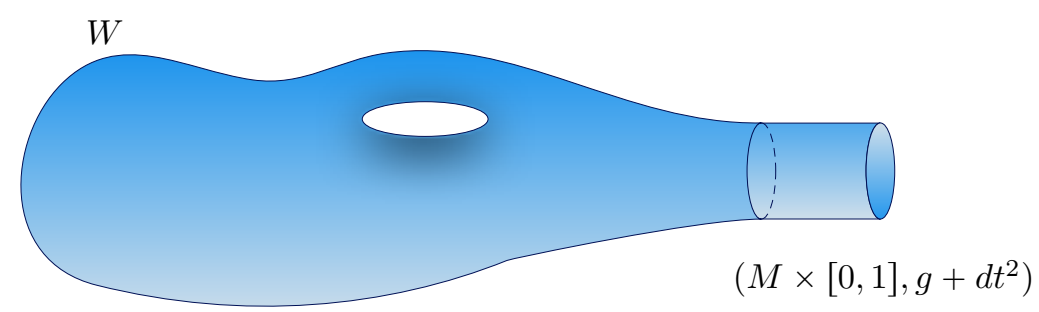

Figure 7. A metric on $W$ which takes the form of a cylindrical product $\left(M \times[0,1], g+d t^{2}\right)$ near the boundary

This survey will be concluded in the next issue of the Bulletin.

\section{REFERENCES}

[1] S. Aloff and N. Wallach, An infinite family of distinct 7-manifolds admitting positively curved Riemannian structures, Bull. Amer. Math. Soc. 81 (1975), 93-97

[2] D. Auckly, H. Jung Kim, P. Melvin and D. Ruberman, Stable isotopy in four dimensions, J London Math Soc (2015) 91 (2): 439-463.

[3] M.F. Atiyay, R. Bott and A. Shapiro, Clifford Modules, Topology 3 Suppl. 1, 1964, 3-38.

[4] I. Belegradek, F. T. Farrell and V. Kapovitch, Spaces of non-negatively curved manifolds and pseuodisotopies, Preprint at arxiv:1501.03475.

[5] I. Belegradek and J. Hu, Connectedness properties of the space of complete non-negatively curved planes, Math. Ann. 362 (2015), no. 3-4, 1273-1286

[6] I. Belegradek, S. Kwasik and R. Schultz, Moduli spaces of non-negative sectional curvature and non-unique souls, J. Diff. Geom. 89 (2011), 49-86

[7] A. L. Besse, Einstein Manifolds, Springer-Verlag (Berlin, 1987)

[8] J.M. Boardman and R. M. Vogt Homotopy Invariant Algebraic Structures on Topological Spaces, Lecture Notes in Mathematics Number 347 (1973)

[9] R. Bott, The stable homotopy of the classical groups, Annals of Mathematics. (1959) Second Series, 70: 313337

[10] B. Botvinnik, Concordance and Isotopy of Metrics with Positive Scalar Curvature, Geom. Funct. Anal. Vol. 23 (2013) 1099-1144

[11] B. Botvinnik, Concordance and Isotopy of Metrics with Positive Scalar Curvature II, arXiv:1604.07466

[12] B. Botvinnik, Johannes Ebert and Oscar Randal-Williams, Infinite Loop Spaces and Positive Scalar Curvature, Invent. math. (2017). doi:10.1007/s00222-017-0719-3

[13] B. Botvinnik, B. Hanke, T. Schick and M. Walsh, Homotopy Groups of the Moduli Space of Metrics of Positive Scalar Curvature, Geometry and Topology 14 (2010) 20472076

[14] B. Botvinnik, M. Walsh and D.J. Wraith The Observer Moduli Space of Metrics of Positive Ricci Curvature in preparation, Summary of Results contained in Oberwolfach Report No. 3/2017 on Mini Workshop: Spaces and Moduli Spaces of Riemannian Metrics 
[15] B. Botvinnik and Ib Madsen, The moduli space of generalized Morse functions, Preprint at arXiv:1004.2895

[16] B. Botvinnik and M. Labbi, Highly connected manifolds of positive pcurvature

To appear in Transactions of the AMS, Preprint: arXiv:1201.1849

[17] B. Botvinnik and P.B. Gilkey, The eta invariant and metrics of positive scalar curvature, Math. Ann 302, no. 3, 1995, 507-517.

[18] R. Carr, Construction of manifolds of positive scalar curvature, Trans. A.M.S. 307, no. 1, May 1988, 63-74.

[19] J. Cerf, La stratification naturelle des espaces de fonctions differentiables reelles et le theoreme de la pseudo-isotopie, Publications mathematiques de l'I.H.E.S., tome 39 (1970), p. 5-173.

[20] S. Chang, Positive Scalar Curvature of Totally Non-Spin Manifolds, Proc. A.M.S. Volume 138, Number 5, May 2010, Pages 1621-1632

[21] V. Chernysh, On the homotopy type of the space $\mathcal{R}^{+}(M)$. Preprint: arXiv 0405235

[22] V. Chernysh, A quasifibration of spaces of positive scalar curvature metrics. Proc. AMS 134, no. 9, September 2006, 2771-2777

[23] F. Coda Marques, Deforming three-manifolds with positive scalar curvature, Annals of Math. Pages 815-863, Volume 176 (2012), Issue 2

[24] R.L. Cohen, A decomposition of the space of generalized Morse functions, Algebraic topology and algebraic K-theory (Princeton, N.J., 1983), 365-391

[25] D. Crowley and T. Schick, The Gromoll filtration, KO-characteristic classes and metrics of positive scalar curvature, Preprint: arXiv 1204.6474

[26] A. Dessai, S. Klaus, W. Tuschmann, Nonconnected moduli spaces of nonnegative sectional curvature metrics on simply-connected manifolds, Bull. London Math. Soc. (to appear).

[27] F. T. Farrell and W. C. Hsiang, On the rational homotopy groups of the diffeomorphism groups of discs, spheres and aspherical manifolds, Proc. Sympos. Pure Math. XXXII AMS, Providence R.I. (1978)

[28] F. T. Farrell and P. Ontaneda, On the topology of the space of negatively curved metrics, J. Diff. Geom. 86, (2010), no. 2, 273-301

[29] T. Friedrichs, Dirac Operators in Riemannian Geometry, AMS Graduate Studies in Mathematics, Volume 25 (2000).

[30] P. Gajer, Riemannian metrics of positive scalar curvature on compact manifolds with boundary, Ann. Global Anal. Geom. 5, no. 3 (1987) 179-191.

[31] S. Galatius, I. Madsen, U. Tilmann and M. Weiss, The homotopy type of the cobordism category, Acta Mathematica, June 2009, Volume 202, Issue 2, pp 195239

[32] S. Galatius and O. Randal-Williams Stable moduli spaces of high-dimensional manifolds, Acta Math., 212 (2) :257377, 2014.

[33] S. Götte, Morse Theory and higher torsion invariants I Preprint, arXiv:math.DG/0111222

[34] A. Gray, The volume of a small geodesic ball of a Riemannian manifold, Michigan Math. J. 20 (1973), 329-344.

[35] M. Gromov, Stable mappings of foliations into manifolds (Russian), Izv. Akad. Nauk SSSR Ser. Mat. 33 (1969), 707-734. English transl., Math. USSRIzv. 3 (1969), 671-694. 
[36] M. Gromov and H. B. Lawson, Jr., The classification of simply-connected manifolds of positive scalar curvature, Ann. of Math. 111 (1980), 423-434.

[37] M. Gromov and H. B. Lawson, Spin and scalar curvature in the presence of a fundamental group I, Ann. Math. 111 (1980), 209-230.

[38] M. Gromov and H. B. Lawson, Jr., Positive scalar curvature and the Dirac operator on complete Riemannian manifolds, Publ. Math. I.H.E.S., no. 58 (1983), 83-196 .

[39] B. Hanke, T. Schick and W. Steimle, The Space of Metrics of Positive Scalar Curvature, Publ. Math. Inst. Hautes Etudes Sci. 120 (2014), 335-367

[40] A. Hatcher and J. Wagoner, Pseudo-Isotopies of Compact Manifolds, Societe Mathematique de France (1973).

[41] A. Hatcher, Algebraic Topology, Cambridge University Press (2002)

[42] M. W. Hirsch, Differential Topology, Springer (1980)

[43] N. Hitchin, Harmonic spinors, Adv. in Math., 14 (1974), 1-55.

[44] S. Hoelzel Surgery stable curvature conditions, Math. Ann. June 2016, Volume 365, Issue 1, pp 1347

[45] K. Igusa, Higher Franz-Reidemeister Torsion, AMS/IP, Studies in Advanced Mathematics, Vol. 31, (2002).

[46] K. Igusa, Higher singularities of smooth functions are unnecessary, Annals of Mathematics, 2nd Ser., Vol. 119, No.1 (Jan., 1984), pp 1-58.

[47] K. Igusa, On the homotopy type of the space of generalized Morse functions, Topology 23 (1994), 245-256.

[48] K. Igusa, The space of framed functions, Trans. A.M.S. 301, no. 2, June 1987, 431-437

[49] K. Igusa, The stability theorem for smooth pseudoisotopies, K-Theory 2 (1988) 1-355

[50] D.D. Joyce, Compact 8-manifoles with holonomy Spin(7), Invent. Math. 123 (1996), no. 3, 507-552.

[51] V. Kapovitch, A. Petrunin and W. Tuschmann, Non-negative pinching, moduli spaces and bundles with infinitely many souls, J. Diff. Geom. 71 (2005) no. 3, 365-383

[52] J.L. Kazdan and F. Warner, Existence and conformal deformation of metrics with prescribed Gaussian and scalar curvature, Ann. of Math. 101 (1975), 317-331.

[53] J.L. Kazdan and F. Warner, Scalar curvature and conformal deformation of Riemannian structure, J. Diff. Geom. 10 (1975), 113-134.

[54] M.A. Kervaire, J.W. Milnor, Groups of homotopy spheres: I, (1963) Annals of Mathematics. Princeton University Press. 77 (3): 504537.

[55] M. Kreck, S. Stolz, Non-connected moduli spaces of positive sectional curvature metrics, J. Am. Math, Soc. 6 (1993), 825-850.

[56] M. Labbi Stability of the p-Curvature Positivity under Surgeries and Manifolds with Positive Einstein Tensor, Annals of Global Analysis and Geometry Volume 15, Number 4 (1997), 299-312.

[57] H.B. Lawson and M.L Michelsohn Spin Geometry, Princeton University Press (1989)

[58] J. Lee, Riemannian manifolds, Springer (1997).

[59] A. Lichnerowicz, Spineurs harmoniques, C. R. Acad. Sci Paris, Ser. A-B 257 (1963), 7-9. 
[60] J. Lohkamp, The space of negative scalar curvature metrics, Invent. Math 110 (1992), no. 2, 403-407.

[61] I. Madsen and M. Weiss, The Stable Moduli Space of Riemann Surfaces: Mumford's Conjecture, Ann. of Math. (2) 165 (2007), no. 3, 843-941

[62] M. Markl, S. Shnider, J. Stasheff, Operads in Algebra, Topology and Physics, Mathematical Surveys and Monographs A.M.S. Vol.96 (2002)

[63] F.C. Marques, Deforming Three-Manifolds with Positive Scalar Curvature, Ann. of Math. Pages 815-863, Volume 176 (2012), Issue 2

[64] P. May, The Geometry of Iterated Loop Spaces, Lecture Notes in Mathematics, Volume 271 (1972)

[65] J. Milnor, Lectures on the h-Cobordism Theorem, Princeton Univ. Press (1965).

[66] Milnor, John W., "On manifolds homeomorphic to the 7-sphere", (1956) Annals of Mathematics, 64 (2): 399405

[67] J. Milnor, Morse Theory, Princeton Univ. Press (1963).

[68] J. Milnor, Remarks concerning spin manifolds, Differential and Combinatorial Topology, a Symposium in Honor of Marston Morse, Princeton Univ. Press (1965), 55-62.

[69] R. S. Palais, Homotopy theory of infinite dimensional manifolds, Topology 5 (1966), 1-16.

[70] N. Perlmutter, Cobordism Categories and Parametrized Morse Theory, Prepint at arXiv:1703.01047

[71] P. Peterson, Riemannian Geometry 2nd Edition, Springer (1998).

[72] A. Ranicki, Algebraic and Geometric Surgery, Oxford University Press (2002).

[73] J. Rosenberg, $C^{*}$-algebras, positive scalar curvature, and the Novikov conjecture, Publ. Math. IHES 58 (1983), 197-212.

[74] J. Rosenberg, $C^{*}$-algebras, positive scalar curvature, and the Novikov conjecture II, Geometric methods in operator algebras (Kyoto, 1983), 341-374, Pitman Res. Notes Math. Ser., 123, Longman Sci. Tech., Harlow, 1986.

[75] J. Rosenberg, $C^{*}$-algebras, positive scalar curvature, and the Novikov conjecture II, Topology 25, no. 3 (1986), 319-336.

[76] J. Rosenberg and S. Stolz, Metrics of positive scalar curvature and connections with surgery, Surveys on Surgery Theory, Vol. 2, Ann. of Math. Studies 149, Princeton Univ. Press, 2001.

[77] D. Ruberman, Positive scalar curvature, diffeomorphisms and the SeibergWitten invariants. Geom. Topol. 5 (2001), 895-924

[78] T. Schick, A counterexample to the (unstable) Gromov-Lawson-Rosenberg Conjecture Topology, Volume 37, Issue 6, 1. November 1998, Pages 11651168

[79] T. Schick, The Topology of Positive Scalar Curvature, Proceedings of the International Congress of Mathematicians Seoul 2014, VOLUME II, 12851308

[80] R. Schoen and S.T. Yau, On the structure of manifolds with positive scalar curvature, Manuscripta Math. 28 (1979), 159-183.

[81] R. Schoen and S.T. Yau, Existence of incompressible minimal surfaces and the topology of three dimensional manifolds of non-negative scalar curvature, Ann. Math. 110 (1979), 127-142. 
[82] Erwin Schrödinger. Diracsches Elektron im Schwerefeld. I. Sitzungsber. Preuss. Akad. Wiss., Phys.-Math. Kl., 1932:105128, 1932.

[83] S. Stolz, Simply connected manifolds with positive scalar curvature, Ann. of Math., 2nd Series 136, no. 3 (1979), 511-540.

[84] S. Stolz, Concordance classes of positive scalar curvature metrics, University of Notre Dame Preprint, http://www.nd.edu/ stolz/preprint.html

[85] C. H. Taubes, The Seiberg-Witten Invariants and Symplectic Forms, Math. Res. Let. 1, (1994), 809-822.

[86] W. Tuschmann, D.J. Wraith, Moduli Spaces of Riemannian Metrics, Oberwolfach Seminars Volume 46 (2015), Birkhäuser

[87] M. Walsh, Metrics of positive scalar curvature and generalised Morse functions, Part 1,

Mem. Am. Math. Soc 209 (2011), no. 983

[88] M. Walsh, Metrics of positive scalar curvature and generalised Morse functions, Part 2,

Trans. Am. Math. Soc. 366 (2014), 150

[89] M. Walsh, Cobordism Invariance of the Homotopy Type of the Space of Positive Scalar Curvature Metrics, Proc. Am. Math. 141 (2013), no. 7, 2475-2484

[90] M. Walsh, H-spaces, loop spaces and positive scalar curvature Geom. Top. 18, (2014), no. 4, 2189-2243

[91] M. Walsh, The space of positive scalar curvature metrics on a manifold with boundary Preprint at arxiv.org

[92] M. Wang, W. Ziller, Einsten metrics with positive scalar curvature Curvature and Topology of Riemannian Manifolds, Proceedings, Lecture Notes in Math. 1021, Springer-Verlag (1986), 319-336

[93] J. Wolfson, Manifolds with k-positive Ricci Curvature, Preprint: www.math.msu.edu/ wolfson/k-positive-Ricci.pdf

[94] D. J. Wraith, Exotic Spheres with Positive Ricci Curvature, J. Diff. Geom. 45 (1997), 638-649.

[95] D. J. Wraith, Surgery on Ricci positive manifolds, J. reine angew. Math. 501 (1998), 99-113.

[96] D. J. Wraith, New connected sums with positive Ricci curvature, Ann. Glob. Anal. Geom. 32 (2007), 343-360

[97] D. J. Wraith, On the moduli space of positive Ricci curvature metrics on homotopy spheres Geom. Top. 15 (2011), 1983-2015

Mark Walsh is a lecturer in Mathematics at Maynooth University. Formerly, he was associate professor of Mathematics at Wichita State University, Kansas, USA. His research interests are in Differential Geometry, in particular the relationship between curvature and topology. He obtained his $\mathrm{PhD}$ from the University of Oregon and a masters and undergraduate degrees from Maynooth University.

(M. Walsh) Department of Mathematics and Statistics, Maynooth University, Maynooth, Co. Kildare, Ireland

E-mail address: Mark.Walsh@mu.ie 